\title{
SYNTHESIS AND ANTINEMATODAL ACTIVITY STUDIES OF SOME FUSED TRIAZINOBENZIMIDAZOLES
}

\author{
Anichina K., PhD, Department of Organic Synthesis and Fuels, University of Chemical Technology \\ and Metallurgy, Sofia, Bulgaria
}

DOI: https://doi.org/10.31435/rsglobal_conf/30122020/7351

Abstract. 4-Aryl-3,4-dihydro[1,3,5]triazino[1,2-a]benzimidazole-2-amines 3a-f were synthesized in the reaction of cyclocondensation between 2-guanidinobenzimidazole and versatile benzaldehydes. Structures of all prepared compounds were confirmed by $I R,{ }^{I} H N M R$ spectroscopy and elemental analysis. Antinematodal activity in vitro of the substances was investigated using isolated Trichinella spiralis muscle larvae. The tested triazonobenzimidazoles showed different activity depending on the substituent $R$ in their molecule as the derivatives substituted with a hydroxyl group demonstrated the best anti-Trichinella spiralis activity in the series.

Keywords: benzimidazoles, triazinobenzimidazoles, antinematodal activity, Trichinella spiralis

Introduction. Parasitic diseases are a global health problem in developing countries with tropical and subtropical climates. In the countries of Eastern Europe, including Bulgaria, regardless of the results achieved in the control of parasitosis, the problem of radical treatment of some of the common intestinal and tissue helminthiasis (e.g. trichinosis, echinococcosis, fasciolosis, filariasis, etc.) is not solved. In clinical practice, drugs from the benimidazole group such as albendazole, mebendazole, thiabendazole and flubendazole are widely used $[1,2]$ but the anthelmintic resistance in parasites is now widespread [3]. For this reason, there is a need for new drugs for the treatment and control of helminth infections. Promising results from pharmacological studies on the anthelmintic activity of benzimidazole derivatives [4-7] determine the increased interest in the synthesis and study of new benzimidazole compounds as potential antinematodal agents. The binding of different pharmacophores into a single molecule would lead to the generation of new compounds, which have a synergistic effect on parasites.

Aim. The aim of the present study is the design and synthesis of compounds combining in one molecule two pharmacophores - benzimidazole and 1,3,5-triazine nucleus, as potential antinematodal agents against the larvae of the Trichinella roundworm.

Materials and Methods. All chemicals were purchased from commercial suppliers. The IR spectra were taken in a $\mathrm{KBr}$ tablet on a Varian apparatus or recorded by ATR on a Bruker Equinox 55 spectrophotometer. ${ }^{1} \mathrm{H}-\mathrm{NMR}$ spectra were taken on a Bruker Avance AV 600 (Bruker, Faelanden, Switzerland). Chemical shifts are expressed in terms of tetramethylsilane (TMS) and are presented in $\delta$ (ppm) using deuterated DMSO as solvent. The microanalyses for $\mathrm{C}, \mathrm{H}$ and $\mathrm{N}$ were performed on PerkinElmer elemental analyzer. Analyses indicated by the symbols of the elements or functions were within $\pm 0.4 \%$ of the theoretical value. All compounds were routinely checked by thin layer chromatography (TLC) using on ALUGRAM SIL G/UV254 pre-coated aluminium sheets with silica gel $60,0.20 \mathrm{~mm}$ thick (Macherey-Nagel, Germany) and the spots were detected under UV light (254 nm).

General procedure for the preparation of 4-aryl-3,4-dihydro[1,3,5]triazino[1,2a]benzimidazole-2-amines: Solution of equimolar amounts of 2-guanidinobenzimidazole $\mathbf{1}$, corresponding benzaldehyde 2a-f and few drops of piperidine in absolute ethanol, were refluxed for 40-60 minutes. After reaction mixture was cooled to the room temperature, the crude product was filtered off and recrystallized from ethanol to obtain products 3a-f.

4-(4-Methoxyphenyl)-3,4-dihydro[1,3,5]triazino[1,2-a]benzimidazole (3a): Yield - 78\%; mp 259-261 ${ }^{\circ} \mathrm{C}$; IR $\left(\mathrm{KBr}, \mathrm{cm}^{-1}\right): v \mathrm{NH}_{2}-3322, \sim 3200 ; v \mathrm{NH}-3102 ; \mathrm{vCH}_{3}-2834,2961 ; v \mathrm{Ar}-1610,1588$, $1510 ; \delta \mathrm{CH}_{3}-1394 ; \nu \mathrm{C}-\mathrm{O}-1247,1027 ; \delta \mathrm{Ar}-832 ; \delta \mathrm{Ar}-\mathrm{bz}-740$. Anal. calcd. for $\mathrm{C}_{16} \mathrm{H}_{15} \mathrm{~N}_{5} \mathrm{O}(\mathrm{C}, \mathrm{H}$, N): Calculated (\%): C, 65.52; H, 5.15; N, 23.88; Found (\%): C, 65.31; H, 5.01; N, 23.76.

4-(4-Fluorophenyl)-3,4-dihydro[1,3,5]triazino[1,2-a]benzimidazol-2-amine (3b): Yield $76 \%$; mp 238-240 ${ }^{\circ}$; IR $\left(\mathrm{KBr}, \mathrm{cm}^{-1}\right): v \mathrm{NH}_{2}-3405,3200 ; v \mathrm{~N}-\mathrm{H}-3121 ; v \mathrm{Ar}-3071 ; \mathrm{vAr}-1648$, 1606,$1510 ; \delta \mathrm{CH}_{3}-1399 ; \delta \mathrm{Ar}-840 ; \delta \mathrm{Ar}-742$. Anal. calcd. for $\mathrm{C}_{15} \mathrm{H}_{12} \mathrm{FN}_{5}(\mathrm{C}, \mathrm{H}, \mathrm{N})$ : Calculated 
(\%): C, 64.05; H, 4.30; N, 24.90; C, 63.98; H, 4.25; N, 24.75; ${ }^{1} \mathrm{H}$ NMR (600 MHz, DMSO) $\delta: 8.25$ $\left(1 \mathrm{H}\right.$, br s, NH), $7.44\left(2 \mathrm{H}, \mathrm{ddd}, J=7.2, J=2.3 \mathrm{~Hz}, \mathrm{H}_{\text {arom }}\right), 7.25\left(1 \mathrm{H}, \mathrm{d}, J=7.5 \mathrm{~Hz}, \mathrm{H}_{\text {arom }}\right), 7.23(2 \mathrm{H}$, ddd, $\left.J=7.2, J=2.3 \mathrm{~Hz}, \mathrm{H}_{\text {arom }}\right), 6.96\left(1 \mathrm{H}, \mathrm{t}, J=7.5 \mathrm{~Hz}, \mathrm{H}_{\text {arom. }}\right), 6.82\left(1 \mathrm{H}, \mathrm{s}, \mathrm{H}_{\text {arom. }}\right), 6.82(1 \mathrm{H}, \mathrm{t}, J=7.5$ $\left.\left.\mathrm{Hz}, \mathrm{H}_{\text {arom. }}\right)\right), 6.76\left(1 \mathrm{H}, \mathrm{d}, J=7.5 \mathrm{~Hz}, \mathrm{H}_{\text {arom. }}\right), 6.65\left(2 \mathrm{H}, \mathrm{s}, \mathrm{NH}_{2}\right)$.

4-(4-Hydroxyphenyl)-3,4-dihydro[1,3,5]triazino[1,2-a]benzimidazol-2-amine (3c): Yield $88 \%$; $\mathrm{mp}>300^{\circ} \mathrm{C}$; IR $\left(\mathrm{KBr}, \mathrm{cm}^{-1}\right): v \mathrm{OH}-3541 ; \mathrm{NH}_{2}-3264, \sim 3200 ; v \mathrm{NH}-3098 ; v \mathrm{Ar}-1632,1583$, $1523 ; v \mathrm{C}-\mathrm{O}-1278 ; \delta \mathrm{OH}-1141 ; \delta \mathrm{Ar}-840,821 ; \delta \mathrm{Ar}-\mathrm{bz}-726$. Anal. calcd. for $\mathrm{C}_{15} \mathrm{H}_{13} \mathrm{~N}_{5} \mathrm{O}(\mathrm{C}, \mathrm{H}, \mathrm{N})$ : Calculated (\%): C, 64.51; H, 4.69; N, 25.07; Found (\%):C, 64.37; H, 4.62; N, 24.91.

4-(3-Methoxyphenyl)-3,4-dihydro[1,3,5]triazino[1,2-a]benzimidazol-2-amine (3d ): Yield 83\%; IR (KBr, $\left.\mathrm{cm}^{-1}\right): v \mathrm{NH}_{2}-3439,3318 ; v \mathrm{NH}-3242 ; \mathrm{vCH}_{3}-2834,2957 ; \mathrm{vAr}-1632,1591,1525$; $\delta \mathrm{CH}_{3}-1404 ; v \mathrm{C}-\mathrm{O}-1252,1043 ; \delta \mathrm{Ar}-704,761 \mathrm{~cm}^{-1} ; \delta \mathrm{Ar}-\mathrm{bz}-742 \mathrm{~cm}^{-1}$; Anal. calcd. for $\mathrm{C}_{16} \mathrm{H}_{15} \mathrm{~N}_{5} \mathrm{O}$ $(\mathrm{C}, \mathrm{H}, \mathrm{N})$ : Calculated (\%): C, 65.52; H, 5.15; N, 23.88; Found (\%): C, 65.36; H, 5.10; N, 23.83. ${ }^{1} \mathrm{H}$ NMR $(600 \mathrm{MHz}, \mathrm{DMSO}) \delta: 8.03(1 \mathrm{H}, \mathrm{bs}, \mathrm{NH}), 7.30\left(1 \mathrm{H}, \mathrm{t}, J=7.9 \mathrm{~Hz}, \mathrm{H}_{\text {arom }}\right), 7.23(1 \mathrm{H}, \mathrm{d}, J=7.9 \mathrm{~Hz}$, $\left.\mathrm{H}_{\text {arom }}\right), 6.96-6.90\left(3 \mathrm{H}, \mathrm{m}, \mathrm{H}_{\text {arom }}\right), 6.86\left(1 \mathrm{H}, \mathrm{d}, J=7.7 \mathrm{~Hz}, \mathrm{H}_{\text {arom }}\right), 6.80\left(2 \mathrm{H}, \mathrm{dd}, J=6.4,3.3 \mathrm{~Hz}, \mathrm{H}_{\text {arom }}\right)$, $6.71\left(1 \mathrm{H}, \mathrm{s}, \mathrm{H}_{\text {arom }}\right), 6.42\left(2 \mathrm{H}, \mathrm{bs}, \mathrm{NH}_{2}\right) ; 3.71\left(3 \mathrm{H}, \mathrm{s}, \mathrm{OCH}_{3}\right)$.

4-(3-Fluorophenyl)-3,4-dihydro[1,3,5]triazino[1,2-a]benzimidazol-2-amine (3e): Yield $75 \%$; mp 274-276 ${ }^{\circ} \mathrm{C}$; IR $\left(\mathrm{KBr}, \mathrm{cm}^{-1}\right): v \mathrm{NH}_{2}-3332,3224 ; v \mathrm{NH}-3142 ; v \mathrm{Ar}-1614,1532,1458 ; \delta \mathrm{Ar}$ - 700, 760; $\delta \mathrm{Ar}-\mathrm{bz}-740$. . Anal. calcd. for $\mathrm{C}_{15} \mathrm{H}_{12} \mathrm{FN}_{5}(\mathrm{C}, \mathrm{H}, \mathrm{N})$ : Calculated (\%): C, 64.05; H, 4.30; $\mathrm{N}, 24.90 ; \mathrm{C}, 63.92 ; \mathrm{H}, 4.23 ; \mathrm{N}, 24.82 ;{ }^{1} \mathrm{H}-\mathrm{NMR}$ (DMSOd-6), $\delta(\mathrm{ppm}): 8.14(1 \mathrm{H}, \mathrm{bs}, \mathrm{NH}), 7.44(1 \mathrm{H}$, td, $\left.J=7.9,2.3 \mathrm{~Hz}, \mathrm{H}_{\text {arom }}\right), 7.24\left(1 \mathrm{H}, \mathrm{d}, J=7.9 \mathrm{~Hz}, \mathrm{H}_{\text {arom }}\right), 7.19\left(2 \mathrm{H} \mathrm{m}, \mathrm{H}_{\text {arom }}\right), 7.14(1 \mathrm{H}, \mathrm{d}, \mathrm{J}=7.9 \mathrm{~Hz}$, $\left.\mathrm{H}_{\text {arom, }}\right), 6.95\left(1 \mathrm{H}, \mathrm{m}, \mathrm{H}_{\text {arom }}\right) ; 6.84\left(2 \mathrm{H}, \mathrm{m}, \mathrm{H}_{\text {arom }}\right) ; 6.80\left(\mathrm{~s}, 1 \mathrm{H}, \mathrm{H}_{\text {arom }}\right) ; 6.51\left(\mathrm{bs}, 2 \mathrm{H}, \mathrm{NH}_{2}\right)$.

4-(3-Hydroxyphenyl)-3,4-dihydro[1,3,5]triazino[1,2-a]benzimidazol-2-amine (3f): Yield $81 \%$; IR $\left(\mathrm{KBr}, \mathrm{cm}^{-1}\right): v \mathrm{OH}-3500 ; v_{\mathrm{NH}_{2}}-3368,3211 ; v \mathrm{Ar}-1618,1530,1461 ; v \mathrm{C}-\mathrm{O}-1253 ; \delta \mathrm{OH}-$ 1106; $\mathrm{Ar}-705,776 ; \delta \mathrm{Ar}-\mathrm{bz}-730$. Anal. calcd. for $\mathrm{C}_{15} \mathrm{H}_{13} \mathrm{~N}_{5} \mathrm{O}(\mathrm{C}, \mathrm{H}, \mathrm{N})$ : Calculated (\%): C, 64.51; H, 4.69; N, 25.07; Found (\%):C, 64.40; H, 4.57; N, 24.89.

\section{Anthelmintic evaluation assay}

The bioassay previously reported by us [4] was used without modifications: encapsulated Trichinella spiralis muscle larvae, 100 specimens per $1 \mathrm{~mL}$ physiological solution were used; the triazinobenzimidazole derivatives tested were dissolved in DMSO and their concentration is shown in Table 1. The samples were incubated in thermostat at a temperature of $37{ }^{\circ} \mathrm{C}$. Control microscopic observation for the viability of Trichinella larvae was performed after 24 and 48 hours.

Results and discussion. The target 4-aryl-3,4-dihydro[1,3,5]triazino[1,2-a]benzimidazole-2amines 3a-f were synthesized by cyclocondensation reaction between the 2-guanidinobenzimidazole 1 and benzaldehydes 2a-f in absolute ethyl alcohol by using piperidine as a catalysts [8] (Scheme 1). The compounds prepared were purified by recrystallization and their chemical structures were confirmed by IR, ${ }^{1} \mathrm{H}$ NMR spectral data.<smiles>N=C(N)Nc1nc2ccccc2[nH]1</smiles>

1<smiles>[184In]</smiles>

2a-f

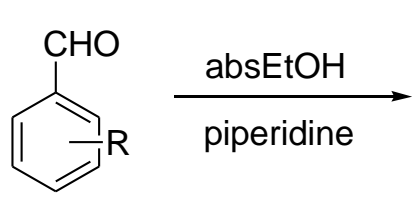

$-f$<smiles>[R]c1ccccc1C1NC(N)=Nc2nc3ccccc3n21</smiles>

3a-f
$\mathrm{R}=4-\mathrm{CH}_{3} ; 4-\mathrm{F} ; 4-\mathrm{OH} ;$

$3-\mathrm{OCH}_{3} ; 3-\mathrm{F} ; 3-\mathrm{OH}$

Scheme 1. Reaction scheme for preparation of 4-aryl-3,4-dihydro[1,3,5]triazino[1,2-a]benzimidazole2-amines $3 a-f$.

All prepared 4-aryl-3,4-dihydro[1,3,5]triazino[1,2-a]benzimidazole-2-amines 3a-f were tested for their antitrichinellosis activities against isolated Trichinella spiralis muscle larvae in the Department of Infectious diseases, Parasitology and Tropical medicine, Medical University, Plovdiv, Bulgaria.

The experimental results, summarized in Table 1, allowed the identification of compound $\mathbf{3 f}$ as the most potent anthelmintic agent (56\% efficacy at a concentration $50 \mu \mathrm{g} / \mathrm{ml}$ after 24 hours). 
Comparing of triazinobenzimidazoles 3a-f, the rank order of anti-trichinellosis efficacy according to the nature of the substituent $\mathrm{R}$ is as follows: $\mathrm{OCH}_{3}<\mathrm{F}<\mathrm{OH}$. The derivatives $\mathbf{3 c}$ and $\mathbf{3 f}$ containing a hydroxyl group in the benzene nucleus $(\mathrm{R}=\mathrm{OH})$ have more pronounced anthelmintic effect than the other compounds. Triazinobenzimidazoles with a substituent at position 3 of the benzene nucleus (3d, $\mathbf{3 e}$ and $\mathbf{3 f})$ are more active than those containing the same substituent at position 4 (3a, $\mathbf{3 b}$ and $\mathbf{3 c}$ ), e.g. 3f $(\mathrm{R}=3-\mathrm{OH})$ is more active than $\mathbf{3 c}(\mathrm{R}=4-\mathrm{OH}), \mathbf{3 e}(\mathrm{R}=3-\mathrm{F})$ is more active than $\mathbf{3 b}(\mathrm{R}=4-\mathrm{F})$. The antinematodal bioassay in vitro showed that tested compounds exhibited higher activity than that of albendazole against Trichinella spiralis larvae.

Table 1 . The in vitro anti-trichinellosis activity of the 4-aryl-3,4-dihydro[1,3,5]triazino[1,2a]benzimidazole-2-amines 3a-f.

\begin{tabular}{|c|c|c|c|c|c|}
\hline \multirow[b]{2}{*}{ Comp. } & \multirow[b]{2}{*}{$\mathrm{R}$} & \multicolumn{2}{|c|}{ Concentration - $50 \mu \mathrm{g} / \mathrm{ml}$} & \multicolumn{2}{|c|}{ Concentration - $100 \mu \mathrm{g} / \mathrm{ml}$} \\
\hline & & $\begin{array}{c}\text { Efficacy after } \\
24 \mathrm{~h}(\%)\end{array}$ & $\begin{array}{c}\text { Efficacy after } \\
48 \mathrm{~h}(\%)\end{array}$ & $\begin{array}{c}\text { Efficacy after } \\
24 \mathrm{~h}(\%)\end{array}$ & $\begin{array}{c}\text { Efficacy after } \\
48 \mathrm{~h}(\%)\end{array}$ \\
\hline $3 \mathrm{a}$ & $4-\mathrm{OCH}_{3}$ & 30.5 & 38.5 & 39.5 & 43.4 \\
\hline $3 b$ & $4-\mathrm{F}$ & 40.2 & 52.4 & 42.9 & 58.8 \\
\hline $3 c$ & $4-\mathrm{OH}$ & 48.6 & 55.8 & 60.4 & 60.5 \\
\hline $3 d$ & $3-\mathrm{OCH}_{3}$ & 36.7 & 55.5 & 44.0 & 58.7 \\
\hline $3 e$ & $3-\mathrm{F}$ & 41.5 & 60.2 & 48.8 & 63.7 \\
\hline $3 f$ & $3-\mathrm{OH}$ & 56.3 & 63.7 & 61.8 & 68.5 \\
\hline \multicolumn{2}{|c|}{ albendazole } & 11.4 & 14.8 & 14.9 & 15.1 \\
\hline
\end{tabular}

Conclusions. 1,3,5-Triazino[1,2-a]benzimidazol-2-amines were prepared by „one pot“ synthesis. The compounds showed moderate in vitro activity against isolated Trichinella spiralis muscle larvae. The results of anthelmintic activity studies may serve as a basis for further planned synthesis of new antihelmintic benzimidazole compounds containing 1,3,5-triazine heterocycle.

Acknowledgments. This study was supported by Grants 11976 by NIS at the University of Chemical Technology and Metallurgy, Sofia, Bulgaria. I would also like to thank Dr. Dimitar Vutchev (Departmant of Infectious diseases, Parasitology and Tropical medicine, Medical University, Plovdiv, Bulgaria) for anthelmintic assays on Trichinella spiralis larvae.

\section{REFERENCES}

1. W.C. Campbell (Ed.), Trichinella and Trichinosis, Plenum Press, USA and London, UK, 1983, 340-355.

2. L. X. Liu, P. F. Weller, Antiparasitic Drugs, N. Engl. J. Med., 334, 1996, 1178-1184.

3. L-F.V. Furtado, A-C. Passos de Paiva Bello, É.M.L. Rabelo, Benzimidazole resistance in helminths: From problem to diagnosis, Acta Tropica, 162, 2016, 95-102.

4. A. Ts. Mavrova, K. K. Anichina, D. I. Vuchev, J. A. Tsenov, M. S. Kondeva, M. K. Micheva. Synthesis and antitrichinellosis activity of some 2-substituted-[1,3]thiazolo[3,2-a]benzimidazol-3(2H)-ones, Bioorg. and Med. Chem., 13, 2005, 5550-5559.

5. A. Ts. Mavrova, K.K. Anichina, D.I. Vuchev, J.A. Tsenov, P.S. Denkova, M.S. Kondeva, M.K. Micheva, Antihelminthic activity of some newly synthesized 5(6)-(un)substituted-1H-benzimidazol-2ylthioacetylpiperazine derivatives, Eur. J. Med. Chem., 2006, 1-9.

6. M. Himaja, B. Sirisha, Moonjit Das, D. Munirajsekhar, Synthesis and anhelmintic activity studies of 1substituted benzimidole derivtve, J. Indian Chem. Soc., 92, 2015, 908-910.

7. A. Mavrova, K. Anichina, O.Izevbekhai, D. Vutchev, G. Popova-Daskalova, D. Yancheva, S. Stoyanov., New 1,3-disubstituted benzimidazol-2-ones as a promising scaffold for the antitrihinellosis agents development, J. Chem. Technol. Metall., 56, 1, 2021, 3-9.

8. M. Hranjec, G. Pavlovic', G. Karminski-Zamola, Synthesis, crystal structure determination and antiproliferative activity of novel 2-amino-4-aryl-4,10-dihydro[1,3,5]triazino[1,2-a]benzimidazoles, J. Mol. Struct., 1007, 2012, 242-251. 